\title{
THE PROBLEMS ASSOCIATED WITH THE MONITORING OF COMPLEX WORKPLACE RADIATION FIELDS AT EUROPEAN HIGH-ENERGY ACCELERATORS AND THERMONUCLEAR FUSION FACILITIES
}

\author{
P. Bilski ${ }^{1}$, J. Blomgren ${ }^{2}$, F. d'Errico ${ }^{3}$, A. Esposito ${ }^{4}$, G. Fehrenbacher ${ }^{5}$, F. Fernàndez ${ }^{6}$, A. Fuchs ${ }^{7}$, N. Golnik ${ }^{8}$, \\ V. Lacoste ${ }^{9}$, A. Leuschner ${ }^{10}$, S. Sandri ${ }^{11}$, M. Silari ${ }^{12, *}$, F. Spurny ${ }^{13}$, B. Wiegel ${ }^{14}$ and P. Wright $^{15}$ \\ ${ }^{1}$ IFJ, Krakow, Poland \\ ${ }^{2}$ TSL/INF, Uppsala University, Sweden \\ ${ }^{3}$ DIMNP, Pisa, Italy \\ ${ }^{4}$ INFN, Frascati, Italy \\ ${ }^{5}$ GSI, Darmstadt, Germany \\ ${ }^{6} \mathrm{UAB}$, Barcelona, Spain \\ ${ }^{7}$ PSI, Villigen, Switzerland \\ ${ }^{8}$ IAE, Swierk, Poland \\ ${ }^{9}$ IRSN, Cadarache, France \\ ${ }^{10}$ DESY, Hamburg, Germany \\ ${ }^{11}$ ENEA, Frascati, Italy \\ ${ }^{12}$ CERN, Geneva, Switzerland \\ ${ }^{13}$ NPI ASCR, Prague, Czech Republic \\ ${ }^{14}$ PTB, Braunschweig, Germany \\ ${ }^{15}$ RAL, Didcot, UK
}

\begin{abstract}
The European Commission is funding within its Sixth Framework Programme a three-year project (2005-2007) called CONRAD, COordinated Network for RAdiation Dosimetry. The organisational framework for this project is provided by the European Radiation Dosimetry Group EURADOS. One task within the CONRAD project, Work Package 6 (WP6), was to provide a report outlining research needs and research activities within Europe to develop new and improved methods and techniques for the characterisation of complex radiation fields at workplaces around high-energy accelerators, but also at the next generation of thermonuclear fusion facilities. The paper provides an overview of the report, which will be available as CERN Yellow Report.
\end{abstract}

\section{INTRODUCTION}

Monitoring of ionising radiation around high-energy particle accelerators is a difficult task due to the complexity of the radiation field. The capability to distinguish between the high- (mostly neutrons) and the low-LET (Linear Energy Transfer) components of the radiation field at workplaces, and to correctly measure them, is of primary importance to evaluate the exposure of personnel. At proton machines, the dose equivalent outside a thick shield is mainly due to neutrons, with some contribution from photons and, to a minor extent, charged particles. At certain locations, the radiation field may contain neutrons with energies exceeding tens of $\mathrm{MeV}$, which contribute 30 to $50 \%$ of the ambient dose equivalent outside the shielding. At high-energy electron accelerators, the dominant secondary radiations are highenergy neutrons, the shielding being thick enough to absorb most of the bremsstrahlung photons.

*Corresponding author: marco.silari@cern.ch
Similar high- and low-LET radiation components are present at experimental nuclear fusion facilities. The nuclear reactions employed - the deuteriumdeuterium (D-D) and the deuterium-tritium (DT) - produce high flux of fast neutrons. The plasma current in the toroidal vessels (tokamak) of fusion experiments based on magnetic confinement, the most practised fusion technology in Europe, generates bremsstrahlung $\mathrm{X}$ rays. Special system components of some fusion facilities, like neutral beam injectors, have their own radiation environment due to neutron and photon fields. Neutron activation for $\mathrm{D}-\mathrm{T}$ based systems like JET is elevated in the invessel components and sometimes it is important also in the material of some associated devices, like in the water cooling system of the ITER project. The resulting radiation fields at workplaces, out of the concrete shielding that encase the main fusion facilities, are dominated by thermal neutrons but fast neutrons and photons are also present.

Neutron and photon dosimetry and spectrometry are thus essential tools in radiation protection 


\section{P. BILSKI ET AL.}

dosimetry around both high-energy particle accelerators and nuclear fusion facilities. There are some similarities between these radiation fields and those encountered at flight altitudes, and it is actually possible to partly 'simulate' the radiation field in the atmosphere with accelerator-produced radiation ${ }^{(1)}$. However, one important difference is that accelerators can operate in pulsed mode so that the radiation fields at workplaces can be pulsed. This is an important aspect to be taken into account for instrument response, and the measurements of average dose equivalent rates for radiation protection purposes in these fields present a challenge for instrumentation.

The European Commission is funding within its Sixth Framework Programme a three-year project (2005-2007) called CONRAD, COordinated Network for RAdiation Dosimetry. The organisational framework for this project is provided by the European Radiation Dosimetry Group EURADOS. One task within the CONRAD project, Work Package 6 (WP6), was to provide a report outlining research needs and research activities within Europe to develop new and improved methods and techniques for the characterisation of complex radiation fields at workplaces around high-energy accelerators, but also at the next generation of thermonuclear fusion facilities.

The CONRAD WP6 report ${ }^{(2)}$ reviews the relevant techniques and instrumentation employed for monitoring neutron and photon fields around high-energy accelerators and fusion facilities (mainly JET and ITER), both in terms of dosimetry and spectrometry, emphasising some recent developments to improve the response of neutron measuring devices beyond $20 \mathrm{MeV}$. The report also reviews the major highenergy European accelerator facilities - both research accelerators and hospital-based hadron therapy centres - and the way workplace monitoring is organised at each of them. On-going research in radiation dosimetry and development work in passive dosimetry and active counting and spectrometric instrumentation at several European laboratories are discussed. Calibration problems are addressed, and the neutron calibration facilities available in Europe are listed. This paper provides a brief overview of the report, focusing in particular on some of the most important issues, such as the influence of the pulsed nature of the radiation field on the instrument and the calibration problems. For the review of the instrumentation and of the European facilities, the reader should refer to ref. (2).

\section{MONITORING OF MIXED RADIATION FIELDS}

Two types of dose quantities exist for radiological protection: body-related 'protection quantities' defined by the International Commission on Radiological Protection (ICRP) ${ }^{(3)}$ and 'operational quantities' defined by the International Commission on Radiation Units and Measurements (ICRU) ${ }^{(4)}$. Although protection quantities serve to define dose limits that are not directly measurable, the exposure can be monitored by calculations or by measuring the operational quantities. Calculations of protection quantities require comprehensive knowledge of the energy and direction distribution of the particles in the radiation field and of their interaction with tissue.

One operational dose quantity suited to demonstrate compliance with the limits of the effective dose at workplaces is the ambient dose equivalent, $H^{*}(10)$, which is the dose equivalent, $H$, at a reference point at $10-\mathrm{mm}$ depth in the ICRU sphere under defined irradiation conditions. Many radiation protection instruments used to measure $H^{*}(10)$ follow measurement principles other than those used in the definition and therefore require calibration with respect to this quantity. An alternative and, in general, more accurate procedure is to measure the spectral neutron fluence and fold this information with an appropriate set of fluence to dose equivalent conversion coefficients. In practice, monitoring instruments usually have a response function, which approximately follows $H^{*}(10)$ for a given type of radiation and over a given energy range. The approaches to the determination of ambient dose equivalent for neutrons are discussed in detail in ICRU Report $66^{(5)}$.

Starting from the beam parameters of the accelerator important to radiation monitoring (type, energy, intensity and time structure of the accelerated particles) or from the characteristics of the radiation produced at nuclear fusion facilities, one can make predictions of the composition of the radiation field outside the shielding and then decide the type of area monitors to be employed (active and/or passive) and how to calibrate them.

\section{PULSED FIELDS AND INSTRUMENT RESPONSE}

Most accelerators operate in pulsed mode. Usually such sources deliver their output pulses in time intervals from nanoseconds to tens of microseconds spaced by at least a few milliseconds. This also concerns most of the conventional electron linacs used in radiotherapy, which are operated at $100-400 \mathrm{~Hz}$ with pulse widths of about $1-10 \mu s$. In some accelerators, the microsecond output pulses consist of a series of separate 'bunches' each of duration of a few picoseconds, although the interval between bunches is generally less than one nanosecond. This time structure within the microsecond pulse can usually 
be ignored for radiation field spectrometry and dosimetry.

Radiation protection at workplaces deals with stray radiation fields outside the shielding. At highenergy accelerators, such radiation fields comprise neutrons, photons and charged particles, with pulses that are usually shorter than $10 \mu$ s with high instantaneous fluence rates and dose rates. Measurements of the average dose equivalent (rate) for radiation protection purpose in these fields present a challenge for instrumentation and may become even more difficult at workplaces in the vicinity of new facilities with increasing particle energy.

At present, the time structure of the stray radiation fields is usually deduced from the design of the accelerator. Little or no experimental work has yet been reported concerning the pulsed structure of the radiation field modified by transport through the shield. It can be expected that thick shields of highenergy accelerators may seriously disturb the initial pulse structure because of e.g. different time of flight of the secondary particles through the material of the shield. The information about the real-time structure behind the shields can be important in order to decide whether a particular radiation field must be considered to be pulsed for a particular dosemeter. An important problem can also be represented by the time structure of high-energy neutron leakage from spallation targets.

The influence of pulsed radiation on the response of radiation detectors is considered in the literature first of all for dosimetry of the primary beam. The guidelines from such studies can be applied in radiation protection at workplaces, but lower dose rates at workplaces comparing with the beam conditions should be taken into account.

The most comprehensive source of information on the dosimetry of pulsed $\mathrm{X}$ ray or electron beams is ICRU Report 34(6). Measurements using ionisation chambers, chemical dosemeters, calorimeters and solid-state devices are discussed. The report provides information on certain precautions to be taken and on the selection of calibration constants needed for dosimetry of pulsed low-LET radiation. High-LET radiation, mainly heavy charged particles and neutrons, is only shortly mentioned in ICRU 34, because there was not enough information about the influence of radiation pulsing on dosimetry in complex radiation fields at the time the report was issued (1982). Some up-to-date information and operational guidelines for radiation protection at particle accelerator facilities with energies from $\sim 5 \mathrm{MeV}$ up to the highest energies available can be found in NCRP Report No. $144^{(7)}$, where the special problems of measurements in pulsed radiation fields are also addressed.

Workplace monitoring in complex radiation fields usually involves instruments based on the use of ionisation chambers, particle counting devices or solid-state detectors. The last two types of detectors are also often used in neutron and charge particle spectrometers. Tissue equivalent proportional counters (TEPC) and recombination ionisation chambers are used for microdosimetry and LET-spectrometry. The influence of the pulsed structure of the particle beam on the instrument response is different for the three classes of detectors.

\section{RADIATION PROTECTION AND MONITORING AT EUROPEAN THERMONUCLEAR FUSION FACILITIES}

Many radiation protection issues at experimental thermonuclear fusion machines and at associated facilities are similar to those arising around medium and low-energy accelerators. Radiation fields around these facilities are complex and mainly consist of neutrons and photons. Pulsed fields, short operation periods, complex operation scenarios and variable radiation energy spectrum are common situations at nuclear fusion facilities. The main difference to the radiation fields at particle accelerators is the lower maximum neutron energy: about $2.5 \mathrm{MeV}$ for $\mathrm{D}-\mathrm{D}$ plasmas and $14 \mathrm{MeV}$ for $\mathrm{D}-\mathrm{T}$ plasmas.

A specific radiation monitoring problem is related to the short time during which the so-called 'plasma burning' (or 'shot' or 'pulse') takes place. In this time period, that ranges from $\sim 1 \mathrm{~s}$ to some tenths of seconds, plasma heating systems are activated and the thermonuclear conditions make the fusion reactions possible. Usually an intense, mixed neutron/ photon radiation field is generated during the burning phase, and to collect the needed dosimetric information, the monitoring response during this interval has to be recorded. This is usually accomplished with active monitors and associated electronic devices suitable to activate the measurement for the time needed and to record the related dosimetric information. A discussion on the radiation monitoring system in use at JET and that planned for ITER is given in ref. (2).

\section{CALIBRATION}

Calibration is the process in which the calibration factor (quotient of the conventional true value by the value indicated) of a measuring device is determined in a reference radiation field of well-known ambient dose equivalent under well-specified calibration conditions ${ }^{(5)}$. Radioactive sources are frequently used, e.g. ${ }^{60} \mathrm{Co}$ or ${ }^{137} \mathrm{Cs}$ sources for photon dosemeters and ${ }^{252} \mathrm{Cf}$ or ${ }^{241} \mathrm{Am}(\mathrm{Be})$ sources for neutron dosemeters, since they can provide stable and reproducible calibration conditions. National standard laboratories, for example, provide such reference fields. Then, if used under conditions 


\section{P. BILSKI ET AL}

identical to the calibration conditions, a calibrated instrument will measure $H^{*}(10)$ correctly. However, under different irradiation conditions, for example in fields of other particle compositions or with other particle energy distributions, deviations will occur since the dosemeters used in radiation protection practice usually do not have ideal response characteristics (e.g. the same energy dependence as the fluence-to-dose equivalent conversion function). In practical applications, these deviations are either small enough for the desired degree of accuracy or the user must apply field-specific correction factors to take the differences between calibration conditions and the conditions actually prevailing into account.

Since the radiation fields at workplaces around high-energy accelerators (but similar considerations apply for the cosmic radiation field in aircrafts responsible for aircrew exposure) differ strongly from those applied in standard calibration, the correction factors required can be large. In addition, since the field characteristics and the response of the instrument to all particles in the field are usually not well known, the correction factors cannot be calculated with the desired precision. The reliability and accuracy in personnel exposure monitoring can therefore be improved by performing the calibration in the field of interest or in a calibration field with similar characteristics. The direct field calibration of instruments in a given workplace requires a reference instrument that should be able to measure the (true value of) ambient dose equivalent (nearly) correctly for all radiation components and energies. The use of reference fields ('simulated workplace fields') produced under laboratory conditions requires particle compositions and spectral fluences similar to those in the workplace of interest. Those fields offer a good opportunity of investigating the dosemeter characteristics and of intercomparing different dosemeters under identical and reproducible conditions.

Photon dosemeters are conventionally calibrated with ${ }^{137} \mathrm{Cs}$ radionuclide sources emitting monoenergetic photon radiation with energy of $0.661 \mathrm{MeV}$. The reference quantity for the calibration is primarily the air kerma, $K_{\mathrm{a}}$, which can be converted to $H^{*}(10)$ by applying appropriate conversion coefficients. Photon dosimetry is mostly understood for pure photon fields as well as low-energy photon spectrometry. In mixed fields, the situation is more complex, as is often not easy to take into account the response of a photon spectrometer or dosemeter to neutrons. On the other hand, photon spectrometry in the high-energy region still needs a lot of development work.

Reference neutron fields can be produced by radionuclide sources, nuclear reactors and nuclear reactions with charged particles from accelerators. A recent review of the subject can be found in ref. (8).
Recommendations for producing reference neutron radiation fields are given by the International Organization for Standardization (ISO) $)^{(9-11)}$. The calibration of neutron instrumentation is discussed in more detail below.

\section{NEUTRON CALIBRATION FIELDS}

The calibration of instruments used for routine neutron monitoring, e.g. rem counters or personal dosemeters, is carried out using reference neutron fields with broad spectral distributions like those produced by radionuclide sources. The spectra encountered at workplaces, however, are usually significantly different from those used for the calibration. Hence, the fluence response $R_{\Phi}(E)$ of the instrument has to be determined as a function of the neutron energy $E$ to enable the calculation of socalled 'field correction factors', which account for the dependence of the response on the neutron spectrum. The experimental determination of the response is carried out using reference fields in which the neutron fluence is concentrated at a single energy (monoenergetic fields) or, at least, the majority of the fluence is at a single energy with only a smaller contribution at other energies (quasimonoenergetic fields). The basic quantity for the specification of reference fields is the spectral neutron fluence $\Phi_{E}$. The neutron ambient dose equivalent $H^{*}(10)$ is obtained from $\Phi_{E}$ by folding the spectral distribution with recommended energydependent fluence-to-dose-equivalent conversion coefficients $h_{\Phi}(E)$.

Monoenergetic or quasi-monoenergetic reference fields are produced by bombarding low-Z targets (D, $\mathrm{T},{ }^{7} \mathrm{Li}$ ) with light ions (protons or deuterons) accelerated with Van-de-Graaff accelerators or cyclotrons. In most cases, monoenergetic neutrons can be obtained only under ideal conditions. In reality, however, the effects of finite target thickness, neutron scattering in the target surroundings and the finite detector size as well as the break-up reactions at higher projectile energies cause deviations from the ideal situation; i.e. the fields are only quasimonoenergetic with a high-energy peak of finite width and a low-energy continuum.

The response of a detector to high-energy neutrons $\left(E_{n}>20 \mathrm{MeV}\right)$ is quite difficult to determine experimentally because of the low-energy tail in the spectrum provided by the available quasi-monoenergetic neutron facilities. Moreover, when measuring in the unshielded radiation fields, the contribution of high-energy hadrons also has to be taken into account (see for example, ref. (12)).

If both the energy and angular response characteristics of an instrument and the energy and direction distribution of the radiation field to be determined are well known-either experimentally or 
theoretically - the response data can be folded with the field data to obtain a field correction factor. An alternative approach is to determine the response of the device either in the radiation field of interest (a field calibration) or in an experimental radiation field of sufficiently similar characteristics (a simulated workplace field). Modern Monte Carlo codes can help a lot in designing instrumentation and in understanding their performances and their response functions to various types of radiation. It is nonetheless important that the simulations are validated with calibration measurements in reference fields. A list of available calibration facilities providing monoenergetic or quasi-monoenergetic beams is given in ref. (2).

\section{SIMULATED WORKPLACE FIELDS}

When selecting a workplace neutron field (designed for calibrating and testing either personal dosemeters or area monitors), one has to consider the characteristics of the field to be simulated (such as its energy and direction distributions) and the response of the instruments or dosemeters used to determine the neutron distributions. Workplace neutron fields can be simulated using three types of irradiation facilities: radionuclide sources, nuclear reactors and particle accelerators ${ }^{(13)}$. Since we are interested in workplace fields around high-energy accelerators, the latter of the three methods is the only practicable one. Essentially only two facilities of this type are available in Europe: the CERF facility at $\mathrm{CERN}^{(1)}$ and CANEL at Cadarache ${ }^{(14)}$.

\section{CONCLUSIONS}

The CONRAD WP6 report has reviewed the principal techniques, based both on the active detectors and passive dosemeters, employed to monitor mixed radiation fields around high-energy particle accelerators and experimental thermonuclear fusion reactors. Neutron measuring devices include rem counters, Bonner sphere spectrometers, bubble detectors and etched track detectors. Techniques discussed for photon dosimetry and spectrometry are scintillation detectors, ionisation chambers, Geiger-Müller counters, TLDs (thermoluminescent dosimeters) and EPR (electron paramagnetic resonance) dosemeters. Instruments capable to distinguish between the lowand high-LET components of a field like TEPCs and recombination chambers are also discussed. Secondary (stray) radiation often keeps 'memory' of the original time structure of the primary beam, and if the beam is made up of very short bursts, the influence of such structure on active instruments has to be properly taken into account when selecting or designing a monitoring system. The characterisation of the neutron field produced at high-energy proton accelerators is quite a challenging task: developments occurred over the past few years to improve the response of neutron counters and spectrometers beyond $20 \mathrm{MeV}$ are discussed.

Instruments and dosemeters used for workplace monitoring usually do not have ideal response characteristics, i.e. the same energy dependence as the fluence-to-dose equivalent conversion function. They are normally employed under irradiation conditions that are different from those in which they were calibrated. Thus deviations will occur and proper correction factors have to be applied.

The response of a device to the various components of a mixed radiation field can nowadays be determined quite precisely by means of Monte Carlo codes. It is nonetheless important that the simulations are validated with calibration measurements in monoenergetic or quasi-monoenergetic reference fields. It is also important to be able to calibrate a dosemeter in a simulated workplace field produced under laboratory conditions with particle compositions and spectral fluences similar to those encountered at the workplace of interest. Such a field offers the opportunity of investigating the dosemeter characteristics and of intercomparing different dosemeters under identical and reproducible conditions.

There are a number of issues that still need to be better understood, such as the problems arising from calibration for high-energy devices; for instance, rem counters with a lead insert, which are also sensitive to low-energy neutrons. For neutrons above $20 \mathrm{MeV}$ only 'quasi-monoenergetic' fields are available, i.e. fields with a major component at one energy, but with an additional broad energy component, usually at lower energies, for which corrections have to be made. In addition, the quasi-monoenergetic neutron fields above $20 \mathrm{MeV}$ are not regularly available for 'routine' calibrations. There is also a certain need of better estimating uncertainties in conversion coefficients.

The basic protection quantity is the effective dose $E$, but for purposes of radiation protection metrology the operational quantity ambient dose equivalent, $H^{*}(10)$, is used, which is meant to be a conservative approximation of $E$. Recent studies ${ }^{(15-17)}$ have shown that in some circumstances, the operational quantities may not always provide an overestimate of protection quantities, so that future developments in instrumentation will have to take this fact into account.

\section{ACKNOWLEDGEMENT}

This work is funded in part by the European Commission under the auspices of the Euratom Sixth Framework Programme for research and training in nuclear energy, Contract No FI6R-012684. 


\section{P. BILSKI ET AL.}

\section{REFERENCES}

1. Mitaroff, A. and Silari, M. The CERN-EU highenergy reference field (CERF) facility for dosimetry at commercial flight altitudes and in space. Radiat. Prot. Dosim. 102, 7-22 (2002).

2. Bilski, P. et al. Complex workplace radiation fields at European high-energy accelerators and thermonuclear fusion facilities. Yellow Report CERN-2006-007, Silari, M. editor (2006).

3. International Commission on Radiological Protection (ICRP). 1990 Recommendations of the International Commission on Radiological Protection. Publication 60 (Oxford: Pergamon Press) (1991).

4. International Commission on Radiation Units and Measurements (ICRU). Quantities and units in radiation protection dosimetry. Report 51 (MD, USA: Bethesda) (1993).

5. International Commission on Radiation Units and Measurements (ICRU). Determination of operational dose equivalent quantities for neutrons. Report 66 (MD, USA: Bethesda) (2001).

6. International Commission on Radiation Units and Measurements (ICRU). The dosimetry of pulsed radiation. Report 34 (MD, USA: Bethesda) (1983).

7. National Council on Radiation Protection and Measurements (NCRP). Radiation protection for particle accelerator facilities. Report 144 (MD, USA: Bethesda) (2003).

8. Schuhmacher, H. Neutron calibration facilities. Radiat. Prot. Dosim. 110, 33-42 (2004).

9. International Organization for Standardization (ISO). Reference neutron radiations-Part 1: characteristics and methods of production. ISO Standard 8529-1 (Geneva: ISO) (2001).
10. International Organization for Standardization (ISO). Reference neutron radiations-Part 2: calibration fundamentals of radiation protection devices related to the basic quantities characterizing the radiation field. ISO Standard 8529-2 (Geneva: ISO) (2000).

11. International Organization for Standardization (ISO). Reference neutron radiations-Part 3: calibration of area and personal dosimeters and determination of their response as a function of neutron energy and angle of incidence. ISO Standard 8529-3 (Geneva: ISO) (1998).

12. Agosteo, S., Dimovasili, E., Fassò, A. and Silari, M. The response of a Bonner sphere spectrometer to charged hadrons. Radiat. Prot. Dosim. 110, 161-168 (2004).

13. International Organization for Standardization (ISO). Reference neutron radiation-Characteristics and method of production of simulated workplace neutron fields. ISO standard 12789-1 (Geneva: ISO) (2000).

14. Gressier, V. Les installations de l'IRSN dédiées à la métrologie des neutrons. IRSN Report DRPH/SDE 2005-012 (2005).

15. ICRP/ICRU Joint Publication. Conversion coefficients for use in radiological protection against external radiation. ICRP Publication 74 (Pergamon: Oxford) (1997) and ICRU Report 57 (MD, USA: Bethesda) (1998).

16. Ferrari, A., Pelliccioni, M. and Pillon, M. Fluence to effective dose conversion coefficients for neutrons up to $10 \mathrm{TeV}$. Radiat. Prot. Dosim. 71, 165-173 (1997).

17. Bartlett, D.T., Drake, P., d'Errico, F., LuszikBhadra, M., Matzke, M. and Tanner, R.J. The importance of the direction distribution of neutron fluence and methods of determination. Nucl. Instrum. Meth. A476, 386-394 (2002). 